\title{
Multi-Objective Optimization of Composite Angle Grid Plates for Maximum Buckling Load and Minimum Weight Using Genetic Algorithms and Neural Networks
}

\author{
Amir Ehsani ${ }^{1}$, Hamid Dalir ${ }^{* 1}$
}

\begin{abstract}
The present work describes an optimization process based on the $\varepsilon$-constraint method to find an optimum design to maximize the critical buckling load and minimize the structural weight of an angle grid plate. A comprehensive geometrical model is considered including all geometrical design variables of the grid. In order to achieve a precise and effective approximation of the buckling load, an artificial neural network (ANN) is employed. Training data for ANN is obtained by the Mindlin plate theory as well as the Ritz method. The ANN is combined with genetic algorithms (GA) to find optimized design variables for the angle grid structure. The results provide a wide range of geometrical data for designers to choose the maximum buckling load at the minimum structural weight.
\end{abstract}

Keywords: Artificial Neural Networks, Angle Grid Structure, Buckling Load, Genetic Algorithm, MultiObjective Optimization, $\varepsilon$-constraint approach

\section{Introduction}

Grid (which is also called lattice) structures are broadly utilized in various structures as an independent member or for stiffening plates and shells. Owing to efficient weight and considerable stiffness, these structures are among the first choices in weight-sensitive applications namely, aviation, automotive, and marine industries. Several known grid patterns are exist, such as, angle grid, isogrid, and orthogrid which can be used for appropriate loading and weight conditions. With increasing importance of low weight structures, conventional materials gradually are substituted with composite materials. Due to their economical fabrication process, and resistance to ambient conditions, fiber reinforced composites have employed as a proper option to manufacture grid structures. Up to now, many research projects are carried out on modeling, manufacturing, and optimization of the grid and grid-stiffened structures.

In multiple previous works, researchers attempted to introduce new classes of grid structures to achieve an improved mechanical responses. These attempts yield to several new structures with specific characteristics namely laminated grids, interlocked grids and curved-ribs grid [1-5]. The majority of studies have paid attention to optimize the composite grid-stiffened or ring-stiffened plates and shells, considering the weight as the primary objective function. Therefore, geometrical parameters and stacking sequences are generally considered as the design variables for performing the optimization process. The second objective functions are mostly stability, free vibration frequency and structural failure probability [6-21]. Depending on several parameters such as number of variables, solution method, and used theories, an optimization problem may take a long

\footnotetext{
${ }^{1}$ Department of Mechanical and Energy Engineering, Purdue School of Engineering and Technology

*Corresponding Author: hdalir@purdue.edu
} 
time. Many researchers employed ANN as a fitting tool to provide a reasonable and applicable proximate results. Generally, due to simplicity and accuracy, GA is the most desirable optimization method in the literature. Combination of GA and ANN has led to a fast and reliable solutions [2226].

Despite the plentiful investigations on optimization of grid plates, to date no published study considers entire geometrical design variables to find an optimal design for angle grid structures.

The current work presents a bi-objective optimization process based on $\varepsilon$-constraint method to simultaneously achieve the maximum critical buckling load at the minimum possible weight of composite angle grid plates. Accordingly, a comprehensive geometrical model of angle grid structures is modeled where the design variables include thickness, width, spaces between and angles of each group of the ribs. The objective functions of the problem are the maximum critical buckling load of the structure and minimizing its weight. To obtain the buckling load of the structure the Ritz method along with Mindlin or first-order shear deformation plate theory (FSDT) is applied. Eight design variables, two objective functions and also the first-order theory cause a large amount of calculations and an extremely time-consuming optimization procedure. Therefore, an ANN is trained by analytical data and used to approximate the results with maximum accuracy and minimum possible time. To find the optimum design among the all possible alternatives the GA is combined with ANN. Since multi-objective optimizations lead to a set of solutions, Paretofrontier curve is depicted to illustrate entire potential designs of the structure.

\section{Problem Definition}

\section{a. The angle grid structure}

Consider a square angle grid plate of side " $a$ ” as illustrated in Fig. 1. Defined plate is assumed to be simply supported along four sides and subjected to a uni-axial compression force. Angle grid structures are composed from two groups of parallel ribs. It is assumed, the thickness, $t$, width, $b$, number, $n$, and orientation, $\Psi$, of each group are potentially can be different from the other one. The ribs are rectangular-shaped cross-section. As is depicted in Fig. 1, the first and second group of ribs are shown by green and blue colors, respectively. $d_{1}$ and $d_{2}$ imply the ribs spacing which is directly related to $n_{1}$ and $n_{2}$ values, respectively.

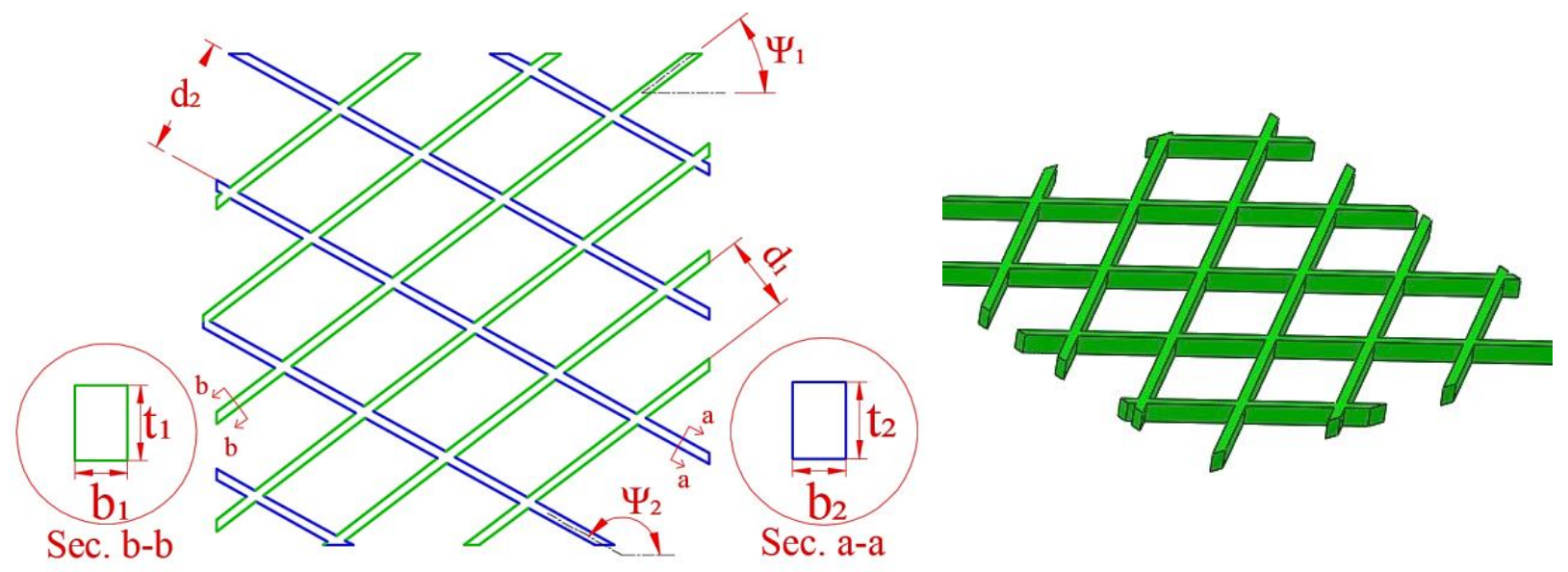

Fig. $12 \mathrm{D} \mathrm{Geometrical} \mathrm{schematic} \mathrm{of} \mathrm{the} \mathrm{defined} \mathrm{angle} \mathrm{grid} \mathrm{structure.} \mathrm{The} \mathrm{first} \mathrm{and} \mathrm{second} \mathrm{group} \mathrm{of} \mathrm{ribs} \mathrm{are} \mathrm{shown}$ by green and blue colors, respectively (left). 3D view of the angle grid structure (right) 
Table 1 shows the domain and other characteristics of each geometrical variable. Both groups of ribs have similar variation domains. However, the orientation angle of first group, $\Psi_{1}$, is considered to change from 0 to 90 degrees related to the $X$-axis while $\Psi_{2}$ can select any integer value between 90 and 180 degrees.

It is assumed that T300/5208 Carbon-Epoxy composite is employed to manufacture the angle grid structure. The elastic properties of the applied material are: $E_{1}=162 \times 10^{9} \mathrm{~Pa}, E_{2}=14.9 \times 10^{9}$ $\mathrm{Pa}, v_{12}=0.283, G_{12}=5.7 \times 10^{9} \mathrm{~Pa}, G_{13}=5.7 \times 10^{9} \mathrm{~Pa}, G_{23}=5.4 \times 10^{9} \mathrm{~Pa}$, and $\rho=1583 \mathrm{~kg} / \mathrm{m}^{3}$ [27]. Where $E_{1}$ and $E_{2}$ are the composite modulus in fiber and transverse to the fiber direction, respectively. $G_{12}, v_{12}$ and $\rho$ are shear modulus, Poisson's ratio and density of the employed composite material, respectively. $G_{13}$ and $G_{23}$ are the out-of-plane shear modulus of the composite. It is also presumed that the ribs are produced by uni-directional fibers along their length.

Table 1 Domains and characteristics of the variables shown in Fig. 1

\begin{tabular}{|c|c|c|c|c|c|}
\hline Variable & Ribs & Description & Symbol & Minimum Value & Maximum Value \\
\hline$x_{1}$ & \multirow{4}{*}{ 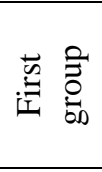 } & number of ribs & $n_{1}$ & 2 & 9 \\
\hline$x_{2}$ & & ribs width & $b_{1}$ & $12.5 \mathrm{~mm}$ & $20 \mathrm{~mm}$ \\
\hline$x_{3}$ & & ribs thickness & $t_{1}$ & $7.5 \mathrm{~mm}$ & $15 \mathrm{~mm}$ \\
\hline$x_{4}$ & & ribs angle & $\Psi_{1}$ & $0^{\circ}$ & $90^{\circ}$ \\
\hline$x_{5}$ & \multirow{4}{*}{ 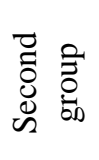 } & number of ribs & $n_{2}$ & 2 & 9 \\
\hline$x_{6}$ & & ribs width & $b_{2}$ & $12.5 \mathrm{~mm}$ & $20 \mathrm{~mm}$ \\
\hline$x_{7}$ & & ribs thickness & $t_{2}$ & $7.5 \mathrm{~mm}$ & $15 \mathrm{~mm}$ \\
\hline$x_{8}$ & & ribs angle & $\Psi_{2}$ & $90^{\circ}$ & $180^{\circ}$ \\
\hline
\end{tabular}

\section{b. Constitutive Equations}

The Mindlin theory or FSDT is utilized to obtain the buckling load of the angle grid structure. According to Mindlin theory, the displacement fields for the given plate is presented in the following form:

$$
u, v, w=u_{0}(x, y)+z \varphi_{x}(x, y), v_{0}(x, y)+z \varphi_{y}(x, y), w(x, y)
$$

In which $u, v$ and $w$ are the displacements along with $x, y$ and $z$ axes. The subscript " 0 " signifies the mid-plane deflections. The $\varphi_{y}$ and $\varphi_{x}$ show rotation angles of transverse normal axis in the midplane surface in $x$ and $y$ axes, respectively. Like a composite ply, a grid plate has the directional properties that is engendered due to the ribs' position and geometries. In this way, the resultant forces, $N$ and $Q$, and moments, $M$, for a symmetrical orthotropic thick plate is written as:

$$
\begin{aligned}
& \begin{array}{ccccccc}
N_{x} & A_{11} & A_{12} & A_{16} & 0 & 0 & u_{0, x} \\
N_{y} & A_{12} & A_{22} & A_{26} & 0 & 0 & v_{0, y} \\
N_{x y}= & A_{16} & A_{26} & A_{66} & 0 & 0 & u_{0, y}+v_{0}
\end{array} \\
& \begin{array}{l}
Q_{y z} \\
\left.Q_{x z}\right\rfloor
\end{array}\left\lfloor\begin{array}{ccccc}
0 & 0 & 0 & A_{44} & A_{45} \\
0 & 0 & 0 & A_{45} & A_{55}
\end{array}\right\rfloor\left\lfloor\begin{array}{l}
\varphi_{y}+w_{, y} \\
\varphi_{x}+w_{, x}
\end{array}\right\rfloor \\
& {\left[\begin{array}{c}
M_{x} \\
M_{y} \\
M_{x y}
\end{array}\right]=\left[\begin{array}{lll}
D_{11} & D_{12} & D_{16} \\
D_{12} & D_{22} & D_{26} \\
D_{16} & D_{26} & D_{66}
\end{array}\right]\left[\begin{array}{c}
\varphi_{x, x} \\
\varphi_{y, y} \\
\varphi_{x, y}+\varphi_{y, x}
\end{array}\right]}
\end{aligned}
$$

Where $[A]$ and $[D]$ matrices are the extensional and bending stiffness matrices respectively and is obtained using the method that is presented by Nemeth [28]. The matrices' elements are a function of material and geometrical properties such as orientation and cross-section of the ribs. 
The Ritz approach is utilized for achieving the critical buckling loads of the plate. Hence, the total potential energy is obtained as below:

$$
\pi=U-V
$$

In which $V$ is loss in the potential energy and $U$ is the strain energy [29, 30].

For a simply supported plate, three trigonometric series can be considered for defining $w(x, y)$, $\varphi_{x}(x, y), \varphi_{y}(x, y)$ in Eq. (1) which satisfy the geometrical boundary conditions of $w, x$ and $y$. They can be expressed as the following set of equations [31]:

$$
w(x, y), \varphi_{x}(x, y), \varphi_{y}(x, y)=\sum_{m=1}^{M} \sum_{n=1}^{N}\left[W_{m n} \sin (X) \sin (Y), P_{m n} \cos (X) \sin (Y), T_{m n} \sin (X) \cos (Y)\right]
$$

Where the $W_{m n}, P_{m n}$, and $T_{m n}$ are the constants and $X, Y$ for a square plate of side " $a$ " are:

$$
X=\frac{m \pi}{a} x, Y=\frac{n \pi}{a} y
$$

Performing a minimization process of the $\pi$ in Eq. (4) respecting to the $W_{m n}, P_{m n}$ and $T_{m n}$ coefficients, produces an eigenvalue problem as below:

$$
\left([K]-\lambda\left[K_{G}\right]\right) \tilde{d}=\tilde{0}
$$

Where $\left[K_{G}\right]$ and $[K]$ are the geometric and elastic stiffness matrices of the angle grid plate. $\tilde{d}$ is the vector of coefficients $\left(W_{m n}, P_{m n}\right.$, and $\left.T_{m n}\right)$ that can be expressed as [30]:

$$
\tilde{d}=\left\{\begin{array}{c}
W_{m n} \\
P_{m n} \\
T_{m n}
\end{array}\right\}
$$

Calculating the $\lambda$ from Eq. (7), leads to the buckling load values.

\section{Artificial Neural Networks}

Artificial Neural Network (ANN) is among the best tools utilized in machine learning. It is engendered according biological nervous systems and works based on training-testing method. ANN is composed of several components namely, input, hidden, and output layers, neurons, and connections. In an ANN multitude associated processing elements, neurons, collaborating to solve a problem. In this way, the network gathers data from previous solved problems to establish an arrangement of neurons that comprehend solving a new examined problem. This method is widely employed to simulate complicated functions with multiple inputs and several outputs which causes a considerable reduction in solving time.

In this study, a Multi-Layers Perceptron (MLP) network with three layers, two hidden and one output layers, are defined (Fig. 2). The training is performed by the Bayesian Regularization (BR) backpropagation training function. In the training procedure, minimization of Mean Square Error (MSE) between the outputs and targets of the training set is considered as the criterion to evaluate the network performance. 


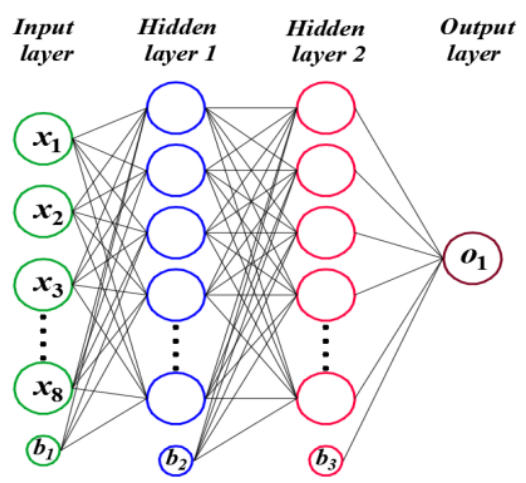

Fig. 2 The neural networks structure. $x_{1}-x_{8}$ are the design variables. $b_{1}-b_{3}$ are biased values. $O_{1}$ is the output which is the buckling load in this study.

The architecture of the ANN employed including the number of neurons and transfer functions of each layer are described in Table 2. A MATLAB code is prepared with association its neural networks toolbox to implement ANN procedure.

Table 2 The architecture of ANN

\begin{tabular}{|c|c|c|c|c|c|c|c|c|}
\hline \multicolumn{2}{|c|}{ First Hidden Layer } & \multicolumn{2}{|c|}{ Second Hidden Layer } & \multicolumn{2}{|c|}{ Output Layer } & $\begin{array}{l}\text { Training } \\
\text { samples }\end{array}$ & $\begin{array}{l}\text { Testing } \\
\text { samples }\end{array}$ & $\begin{array}{c}\text { Validating } \\
\text { samples }\end{array}$ \\
\hline neurons & Transfer fn. & neurons & Transfer fn. & neurons & Transfer fn. & & & \\
\hline 11 & $\begin{array}{l}\text { Logarithmic } \\
\text { sigmoid }\end{array}$ & 11 & $\begin{array}{l}\text { Tangent } \\
\text { sigmoid }\end{array}$ & 1 & Linear & $70 \%$ & $15 \%$ & $15 \%$ \\
\hline
\end{tabular}

\section{Multi-Objective Optimization}

\section{a. Optimization formulation}

In contrary with single-objective optimization problems, generally, there is no unique solution for multi-objective optimization due to a trade-off among the objectives. Therefore, results of these problems are typically presented by a Pareto-frontier curve, which is a set of optimal solutions [32].

In the current study, the multi-objective optimization of an angle grid plate is implemented. The objective functions are the total weight and the critical buckling load of the structure. Eight geometrical parameters are considered as design variables namely, thickness, width, number and angle of each group of ribs (see Table 1).

The $\varepsilon$-constraint approach is employed to achieve the optimal interaction between minimum weight and maximum critical buckling load of the structure. In this method, only one of the defined objectives is optimized, $f_{n}(\boldsymbol{x})$, and the rest of the objectives, $f_{k}(\boldsymbol{x})$, is imposed to the problem as the constraints [33]. Consequently, a multi-objective problem converts to a single-objective optimization with one or more new constraints. Using $\varepsilon$-constraint method, the optimization problem is solved for any given upper bound of $\varepsilon$ to find $f_{n}(\boldsymbol{x})$. In this way, the Pareto-frontier curve can be drawn to illustrate the optimal design space. For $K$ number of objectives the method can be expressed as below:

$$
f_{k}(\boldsymbol{x}) \leq \varepsilon_{k}, \quad k=1, \ldots, K ; \quad k \neq n
$$

Where $\varepsilon_{k}$ is an upper limit for objective function $f_{k}(\boldsymbol{x})$ and $\boldsymbol{x}$ represents a vector of inputs. 
In the present work, the critical axial buckling load is considered as the main objective function and the structure's weight is applied as the $\varepsilon$-constraint. Increasing the $\varepsilon$ value gradually and resolve the optimization problem leads to a maximum buckling load for each weight step and subsequently the optimal design space is generated. Therefore the optimization problem is formulated as follows:

$$
\begin{aligned}
& \text { Maximize } \quad f_{n}(\boldsymbol{x})=N_{c r}\left(x_{1}, x_{2}, \cdots, x_{8}\right) \\
& \text { Subjected to } \quad f_{k}(\boldsymbol{x})=W\left(x_{1}, x_{2}, \cdots, x_{8}\right) \leq \varepsilon_{k} ; W_{\min } \leq \varepsilon_{k} \leq W_{\max } ; k \neq n \text {; } \\
& 2 \leq x_{1}, x_{5} \leq 9 \\
& 12.5 \mathrm{~mm} \leq x_{2}, x_{6} \leq 20 \mathrm{~mm} \\
& 7.5 \mathrm{~mm} \leq x_{3}, x_{7} \leq 15 \mathrm{~mm} \\
& 0^{\circ} \leq x_{4} \leq 90^{\circ} \\
& 90^{\circ} \leq x_{8} \leq 180^{\circ}
\end{aligned}
$$

In which $N_{c r}$ is the critical buckling load objective function. $W$ is the weight function, $W_{\min }$ and $W_{\max }$ are the minimum and maximum feasible weights, respectively.

\section{b. Genetic algorithm}

GA is a searching approach to identify the superior members of a generation and transfer to the later one. Moreover, genetic algorithm is a proper method for discrete optimization problems [9]. Genetic algorithm has been chosen to search the feasible objectives space. In fact, for every upper limit of weight, $\varepsilon_{k}$, GA searches among possible design variable domains to find an optimal solution for $N_{c r}(\boldsymbol{x})$.

As illustrated in Table 1, the eight geometrical parameters are considered as the design variables. The selected variables are completely independent and can change in their defined domains. Weight of the angle grid is taken as the problem constraints. The critical buckling load is the fitness function. In addition, a penalty value is deducted from the objective function to penalize infeasible solutions for weakening their fitness values. Therefore, the objective function in Eq. (10) can be reformed to the following fitness function:

$$
\text { fit }_{n}(\boldsymbol{x})=f_{n}(\boldsymbol{x})-\delta_{k} C_{k}=N_{c r}\left(x_{1}, x_{2}, \cdots, x_{8}\right)-\delta_{k} C_{k} \quad\left\{\begin{array}{c}
\delta_{k}=1, \text { if } f_{k}(\boldsymbol{x}) \text { is violated } \\
\delta_{k}=0, \text { for any other cases }
\end{array}\right.
$$

Where $C_{k}$ implies the penalty coefficient constant.

In a chromosome, the all design variables of the angle grid, $\left(x_{1} \ldots x_{8}\right)$, are encoded as a binary form. The first population for genetic algorithm process is randomly produced. Three genetic operators namely, selection, crossover, and mutation are employed to procreate the new population (offspring) from the current population (parents).

For selection operator, Tournament and Elitist mechanisms are utilized for generating the $20 \%$ of the next generation. The mutation operator is employed to replace a random gen from an arbitrary chromosomes from 1 to 0 or vice versa to expand the diversity of generations. The crossover operator is applied to interchange and integrate genes between two chromosomes. Due to the long chromosome string with vast amount of information about the design variables a six-point crossover is used to reproduce $75 \%$ of the children from a pair of random parents.

To perform the optimization process a MATLAB subroutine has been written for GA and combined with corresponding code for ANN. 
Since the maximum fitness amounts were not specified, the GA was implemented several times with various number of generations and populations to find the optimum values. Evaluating the convergence of the GA, an optimum number of generations is considered as the completion criterion. In the Appendix, Fig. A1 depicts the changes of the maximum critical load in various generations for several random weight steps. Evidently, after about one hundred generations, the GA is converged to the desired value. Table 3 illustrates the GA tuned parameters.

Table 3 The GA parameters

$\begin{array}{cc}\text { Number of Population } & 250 \\ \text { Selection } & 20 \% \\ \text { Crossover } & 75 \% \\ \text { Mutation } & 5 \% \\ \text { Completion } & 150 \text { generations }\end{array}$

\section{Results and Discussion}

The relation between the both objective functions is shown in Fig. 3. The critical buckling loads are normalized with respect to the maximum achieved load and it is represented as, $\tilde{N}_{c r}$. Two areas are specified in the figure. First one is the feasible objectives space, which illustrates all the possible solutions for the design space. In this area, every point has a special input vector and two objective values, weight and buckling. The GA is searched this area to find the prominent members of the input vectors to have the maximum critical buckling load at the minimum given weight. Among the all solutions, Pareto-frontier curve shows the optimal solutions for the optimization problem. Each point of this curve defines a design variables vector, $\left(x_{1} \ldots x_{8}\right)$, which in its turn has the maximum critical buckling load for a given weight. According to the application and design priorities, each point of the curve can be a solution for the multi-objective optimization problem.

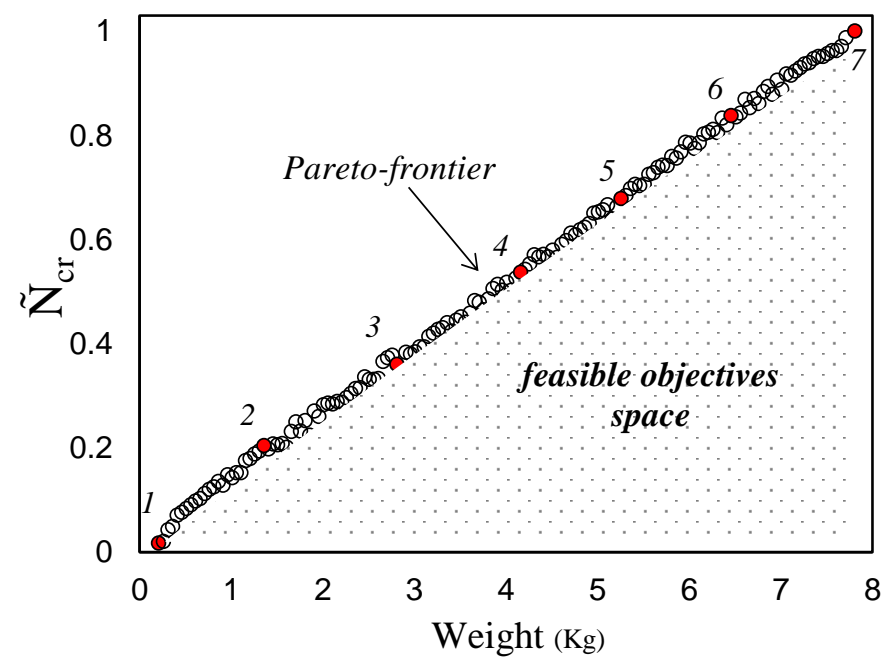

Fig. 3 Feasible objectives space for all input vectors, $\left(x_{1} \ldots x_{8}\right)$ and Pareto-frontier curve

As can be seen in Fig. 3, the Pareto-frontier follows a linear trend. However, linearity does not mean that increasing the weight causes the optimized buckling load. Many points in the feasible objectives space have high weight with low critical buckling load.

Seven arbitrary points are specified on the Pareto-frontier curve with red color and a number. The input vectors of these cases are extracted and depicted in Table 4. Under the design variables, the 
domain of each parameter is also mentioned. On the right hand of the table weight, approximate and analytical buckling load and their discrepancy values are listed. As Table 4 shows, approximate results are in acceptable agreement with analytical results.

Table 4 Input vectors and the value of objective functions for seven specified cases in Fig. 3

\begin{tabular}{|c|c|c|c|c|c|c|c|c|c|c|c|c|}
\hline $\begin{array}{l}\stackrel{\text { Variables }}{\rightarrow} \\
\text { Cases } \downarrow\end{array}$ & $\begin{array}{c}x_{I} \\
(2-9)\end{array}$ & $\begin{array}{c}x_{2} \\
(12.5-20)\end{array}$ & $\begin{array}{c}x_{3} \\
(7.5-15)\end{array}$ & $\begin{array}{c}x_{4} \\
(0-90)\end{array}$ & $\begin{array}{c}x_{5} \\
(2-9)\end{array}$ & $\begin{array}{c}x_{6} \\
(12.5-20)\end{array}$ & $\begin{array}{c}x_{7} \\
(7.5-15)\end{array}$ & $\begin{array}{c}x_{8} \\
(90-180)\end{array}$ & $W_{(k g)}$ & $\begin{array}{c}\tilde{N} c r \\
(A N N)\end{array}$ & $\begin{array}{c}\tilde{N} c r \\
(F S D T)\end{array}$ & Err. \\
\hline 1 & 2 & 12.5 & 11 & 48 & 2 & 12.5 & 7.5 & 136 & 0.212 & 0.016 & 0.017 & 1.057 \\
\hline 2 & 4 & 12.5 & 15 & 44 & 3 & 18 & 15 & 150 & 1.362 & 0.204 & 0.216 & 5.268 \\
\hline 3 & 9 & 12.5 & 15 & 48 & 3 & 19.5 & 15 & 137 & 2.812 & 0.360 & 0.351 & 2.620 \\
\hline 4 & 8 & 12.5 & 15 & 45 & 9 & 13 & 15 & 169 & 4.162 & 0.537 & 0.514 & 4.479 \\
\hline 5 & 9 & 13 & 11 & 48 & 9 & 16 & 15 & 129 & 5.262 & 0.678 & 0.646 & 4.996 \\
\hline 6 & 9 & 18 & 15 & 48 & 9 & 16.5 & 15 & 135 & 6.462 & 0.838 & 0.810 & 3.357 \\
\hline 7 & 9 & 20 & 15 & 45 & 9 & 20 & 15 & 135 & 7.810 & 1.000 & 1.002 & 0.203 \\
\hline
\end{tabular}

Considering Table 4 illustrates, both the $4^{\text {th }}$ and $5^{\text {th }}$ cases have 8 and 9 ribs in each stiffeners group as well as minimum width. This shows, comparing to enlarging the width of the ribs, increasing their numbers has more positive effects on the buckling load.

Fig. 4 represents the scatter plot of the design variables versus the normalized buckling load, $\tilde{N}_{c r}$, for all points on the Pareto-frontier curve.

Evaluating the thickness of the ribs $\left(x_{3}, x_{7}\right)$ in Fig. 4 (a and b) and Table 4, demonstrates nearly all cases take the maximum feasible value in the domain. It can be concluded, the thickness of the ribs is a crucial parameter for achieving maximum buckling load in a given weight and the structures sacrifice other design variables to increase its thickness as much as possible.

As shown in Fig. 4 ( $c$ and d) as well as Table 4 the width of the ribs $\left(x_{2}, x_{6}\right)$ are diverse among the optimum cases. However, $b_{1}$ varies less than $b_{2}$. Therefore, it can be considered that the $b_{1}$ is less important than $b_{2}$ since, for low amounts of $\tilde{N}_{c r}$, the GA generally selects minimum values of $b_{1}$ to keep the weight down while increasing $b_{2}$ and the number of ribs $n_{1}$ and $n_{2}$. This shows the width of the ribs is the most convenient variable that can be changed to find the optimum weight and first buckling load of the angle grid structure.

Similar to width of the ribs, number of ribs in each group is variant for the different buckling load values (see Fig. 4 (e and f)). However, more attention shows that 9 number of the ribs are more frequent than others. This can be figured out that more number of ribs even with lower weight and width causes higher buckling load for the angle grid plate. Furthermore, as shown in the plots, $n_{2}$ after $\tilde{N}_{c r}=0.4$, generally reaches to its maximum feasible value, while the width of the corresponding ribs rarely have its upper bound values in this limit.

As can be seen in Fig. 4 ( $\mathrm{g}$ and h), similar to the thickness values, the ribs orientations, $\Psi_{1}$ and $\Psi_{2}$, generally take a value in the range $\left(45^{\circ} \pm 3^{\circ}\right)$ and $\left(135^{\circ} \pm 3^{\circ}\right)$, respectively. This means the optimum condition of the ribs happens when they are perpendicular to each other. If two groups of ribs are placed in an orthogonal situation related to one another, the angle grid turns to an Orthogrid structure. It can be inferred that an orthogrid plat which is wholly rotated about 45 degrees can be substituted by an angle grid with different orientations for each ribs. 


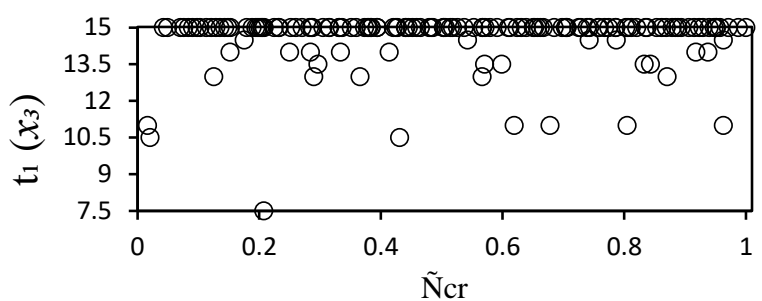

(a)

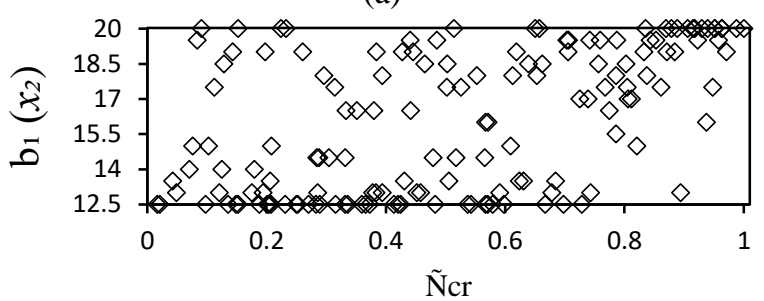

(c)

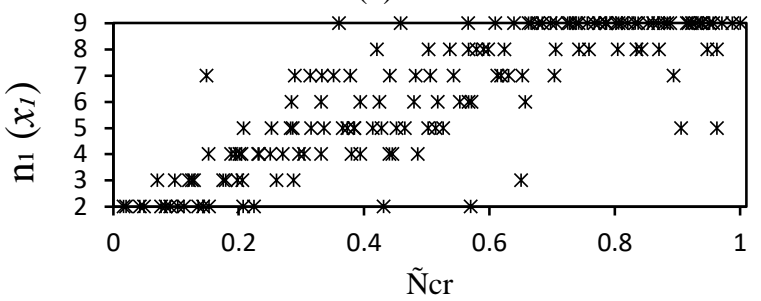

(e)

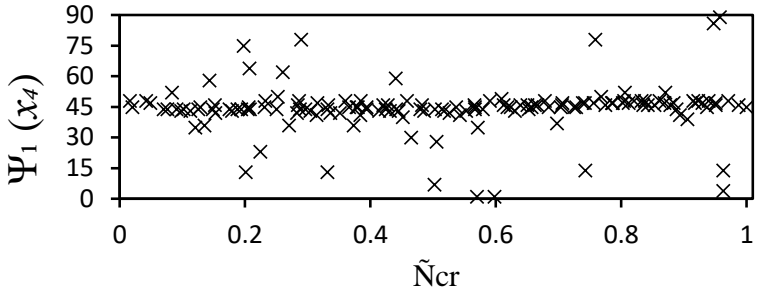

(g)

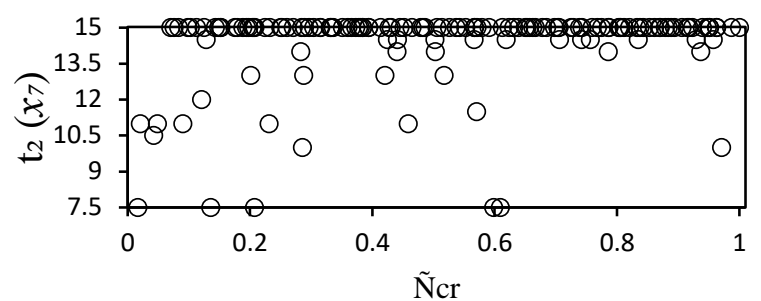

(b)

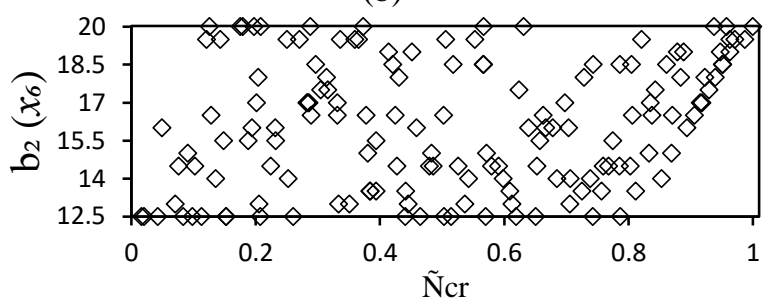

(d)

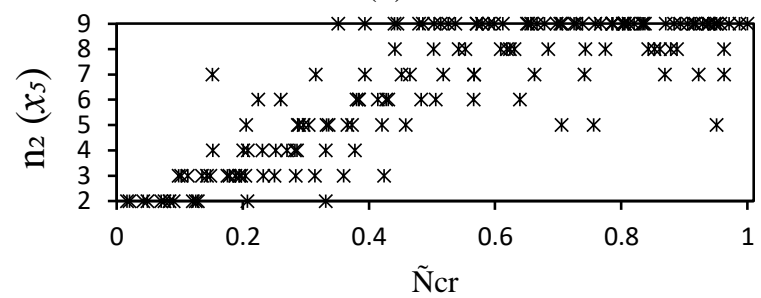

(f)

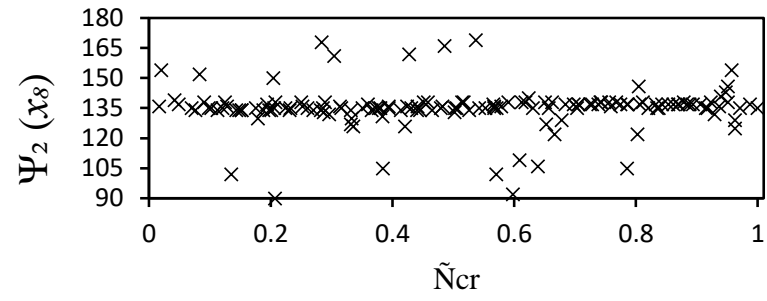

(h)

Fig. 4 Scatter plot of the design variables versus normalized critical buckling load for all points on the Paretofrontier curve in Fig. 3

Fig. 5 illustrates the total cross-section area of the first and second groups of the ribs versus normalized critical buckling load. The total areas are obtained as follows:

$$
A_{1}, A_{2}=\left(n_{1} \times t_{1} \times b_{1}\right),\left(n_{2} \times t_{2} \times b_{2}\right)
$$

As can be seen in Fig. 5, typically increasing the cross-section areas the buckling load increases. Moreover, the points are more inclined to $A_{2}$ axis which it proves previous inference about the role of second groups of ribs which was Increasing the $A_{2}$ values has more influence on the critical buckling load.

Considering the variations of the design variables versus first buckling load it can be concluded that the maximum buckling load values normally happen when the ribs' thicknesses are in upper bound of their domain and the ribs' orientations, $\Psi_{1}$ and $\Psi_{2}$, have a value in the range $\left(45^{\circ} \pm 3^{\circ}\right)$ and $\left(135^{\circ} \pm 3^{\circ}\right)$, respectively. This means an Orthogrid plate with constant thickness and a $45^{\circ}$ rotation respect to the $X$-axis can be substituted by an angle grid. 
Furthermore, results show the number of ribs and the ribs' width are generally varied to find the optimum buckling load at a given weight. Between former variables, $b_{1}$ and $b_{2}$ show more flexibility to change among different optimum solutions.

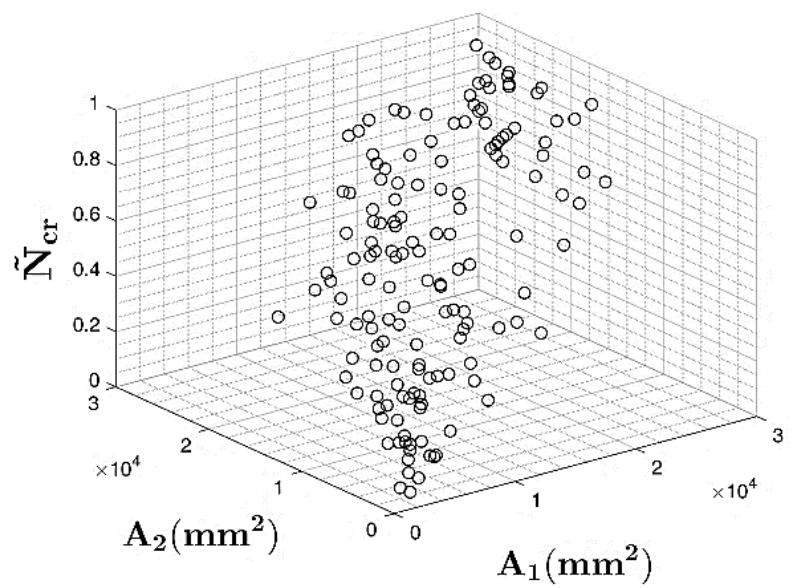

Fig. 5 The total cross-section areas of the first and second groups of the ribs $A_{1}, A_{2}$ versus normalized critical buckling load $\tilde{N}_{c r}$ or all points on the Pareto-frontier curve in Fig. 3

\section{Conclusion}

In the current research, the Multi-objective optimization of an angle grid plate is implemented considering two objective functions, maximum critical buckling load, and minimum weight. Eight design variables including, thickness, width and orientation of the ribs, along as the number of ribs are taken into account. Mindlin plate theory and Ritz method is employed to calculate the first buckling load of the plate. An artificial neural network is used to simulate the solving process and reduce the solving time. Utilizing the GA method along with $\varepsilon$-constraint approach, the maximum critical buckling load for each given weight is obtained and Pareto-frontier curve is drawn.

Results demonstrate the ribs' thickness and orientations tend to reach determined values during optimization process. In contrary, the ribs' width and also the number of ribs vary among the optimum solutions. Furthermore, the values of rib's orientation signify the best performance happens when they are perpendicular to each other and both are in the middle of their domains. Moreover, results describe almost all optimum points on the Pareto-frontier curve have the maximum thickness which expresses the importance of the thickness related to the other design variables.

\section{References}

[1] Han D, Tsai SW. Interlocked Composite Grids Design and Manufacturing. Journal of Composite Materials. 2003;37:287-316.

[2] Wujun C, Gongyi F, Jinghai G, Yanli H, Shilin D. A new design conception for large span deployable flat grid structures. International Journal of Space Structures. 2002;17:293-9.

[3] Ehsani A, Rezaeepazhand J. Vibration and stability of laminated composite orthogrid plates. Journal of Reinforced Plastics and Composites. 2016;35:1051-61.

[4] Wang D, Abdalla MM, Zhang W. Buckling optimization design of curved stiffeners for grid-stiffened composite structures. Composite Structures. 2017;159:656-66.

[5] Ehsani A, Rezaeepazhand J. Fabrication of Laminated Composite Grid Structures using VIP. Fibers and Polymers. 2019;Accepted for Publication. 
[6] Gürdal Z, Gendron G. Optimal design of geodesically stiffened composite cylindrical shells. Composites Engineering. 1993;3:1131-47.

[7] Ambur DR, Jaunky N. Optimal design of grid-stiffened panels and shells with variable curvature. Composite Structures. 2001;52:173-80.

[8] Belardi VG, Fanelli P, Vivio F. Structural analysis and optimization of anisogrid composite lattice cylindrical shells. Composites Part B: Engineering. 2018;139:203-15.

[9] Ehsani A, Rezaeepazhand J. Stacking sequence optimization of laminated composite grid plates for maximum buckling load using genetic algorithm. International Journal of Mechanical Sciences. 2016;119:97-106.

[10] Ambur DR, Jaunky N, Hilburger M, Dávila CG. Progressive failure analyses of compression-loaded composite curved panels with and without cutouts. Composite Structures. 2004;65:143-55.

[11] Bagheri M, Jafari AA, Sadeghifar M. Multi-objective optimization of ring stiffened cylindrical shells using a genetic algorithm. Journal of Sound and Vibration. 2011;330:374-84.

[12] Zhang S, Liu Y, Li W. Optimization on acoustic radiation measuring points of underwater stiffened cylindrical shell based on acoustic transfer vector. IEEE; 2016. p. 134-8.

[13] Ehsani A, Dalir H. Influence of Employing Laminated Isogrid Configuration on Mechanical Behavior of Grid Structures. Journal of Reinforced Plastics and Composites. 2019;Accepted for Publication.

[14] Iuspa L, Ruocco E. Optimum topological design of simply supported composite stiffened panels via genetic algorithms. Computers \& Structures. 2008;86:1718-37.

[15] Wang W, Guo S, Chang N, Yang W. Optimum buckling design of composite stiffened panels using ant colony algorithm. Composite Structures. 2010;92:712-9.

[16] Ehsani A, Dalir H. Tip Angle Effect on Buckling and Deformation of Laminated Composite Anglegrid Plates. 18th European Conference on Composite Materials (ECCM 18). Athens, Greece2018.

[17] Kristinsdottir BP, Zabinsky ZB, Tuttle ME, Neogi S. Optimal design of large composite panels with varying loads. Composite Structures. 2001;51:93-102.

[18] Nagendra S, Jestin D, Gürdal Z, Haftka RT, Watson LT. Improved genetic algorithm for the design of stiffened composite panels. Computers \& Structures. 1996;58:543-55.

[19] Ehsani A, Dalir H. Application of Laminated Composite Grids as a Reinforcing Element for Automotive Components. 18th US-Japan Conference on Composite Materials. Seattle, Washington, USA, 2018.

[20] Kang J-H, Kim C-G. Minimum-weight design of compressively loaded composite plates and stiffened panels for postbuckling strength by genetic algorithm. Composite Structures. 2005;69:239-46.

[21] Ehsani A, Rezaeepazhand J. Comparison of Stiffness and Failure Behavior of the Laminated Grid and Orthogrid Plates. Journal of Solid Mechanics. 2017:126-37.

[22] Marín L, Trias D, Badalló P, Rus G, Mayugo JA. Optimization of composite stiffened panels under mechanical and hygrothermal loads using neural networks and genetic algorithms. Composite Structures. 2012;94:3321-6.

[23] Fu X, Ricci S, Bisagni C. Minimum-weight design for three dimensional woven composite stiffened panels using neural networks and genetic algorithms. Composite Structures. 2015;134:708-15.

[24] Lanzi L, Bisagni C. Minimum weight optimization of composite stiffened panels using neural networks. Structures, Structural Dynamics, and Materials Conference. Norfolk, Virginia. 2003; . p. 1698.

[25] Gholizadeh S, Salajegheh E, Torkzadeh P. Structural optimization with frequency constraints by genetic algorithm using wavelet radial basis function neural network. Journal of Sound and Vibration. 2008;312:316-31.

[26] Lanzi L, Giavotto V. Post-buckling optimization of composite stiffened panels: computations and experiments. Composite Structures. 2006;73:208-20.

[27] Naik NK, Chandra Sekher Y, Meduri S. Damage in woven-fabric composites subjected to low-velocity impact. Composites Science and Technology. 2000;60:731-44.

[28] Nemeth MP. A Treatise on Equivalent-Plate Stiffnesses for Stiffened Laminated-Composite Plates and Plate-Like Lattices. NASA TP 2011-216882 2011. 
[29] Reddy JN. A penalty plate-bending element for the analysis of laminated anisotropic composite plates. International Journal for Numerical Methods in Engineering. 1980;15:1187-206.

[30] Dawe DJ, Craig TJ. The vibration and stability of symmetrically-laminated composite rectangular plates subjected to in-plane stresses. Composite Structures. 1986;5:281-307.

[31] Reddy JN. Mechanics of Laminated Composite Plates and Shells: Theory and Analysis. 2 ed: CRC Press, 2003.

[32] Tamaki H, Kita H, Kobayashi S. Multi-objective optimization by genetic algorithms: A review. IEEE international conference on evolutionary computation: IEEE; 1996. p. 517-22.

[33] Deb K. Multi-Objective Optimization Using Evolutionary Algorithms. 1 ed: Wiley, 2009.

\section{Appendix}

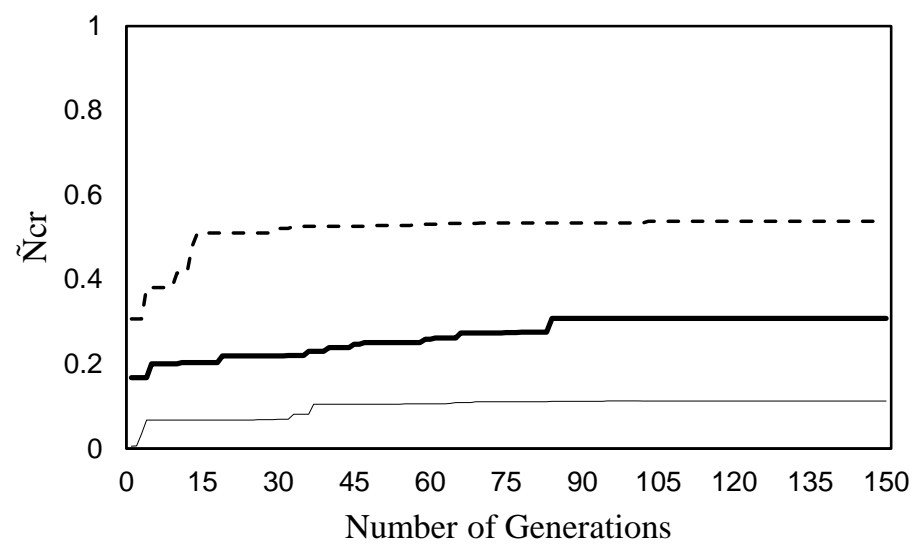

Fig. 1A The critical buckling load changes in various generations for three random weight steps 